\title{
Maraba Oncolytic Virus Expressing Mutant HPV E6/E7
}

National Cancer Institute

\section{Source}

National Cancer Institute. Maraba Oncolytic Virus Expressing Mutant HPV E6/E7. NCI

Thesaurus. Code C157776.

A cancer vaccine comprised of a recombinant, attenuated form of the oncolytic rhabdovirus Maraba (MG1) encoding inactive, mutant forms of the human papillomavirus (HPV) transforming proteins E6 and E7, with potential immunostimulating and antineoplastic activities. Upon administration of MG1-E6E7, MG1 preferentially infects tumor cells and induces the expression of the E6 and E7 proteins. The MG1 virus exerts its oncolytic activity, thereby directly lysing tumor cells. Following the lysis of infected cells, the virus is released and can infect adjacent cells, which both induces further tumor cell oncolysis and may activate the immune system to kill the infected tumor cells. The expressed E6 and E7 proteins stimulate the host immune system to mount a cytotoxic T-lymphocyte (CTL) response against tumor cells expressing HPV E6 and E7, thereby further inducing tumor cell lysis. Oncoproteins E6 and E7 play a key role in the development of cervical intraepithelial neoplasia (CIN) and cervical carcinoma. 